M. Morishita and T. Watanabe

Nagoya Math. J.

Vol. 143 (1996), 111-117

\title{
A NOTE ON THE MEAN VALUE THEOREM FOR SPECIAL HOMOGENEOUS SPACES
}

\author{
MASANORI MORISHITA AND TAKAO WATANABE
}

\section{Introduction}

Let $G$ be a connected linear algebraic group and $X$ an algebraic variety, both defined over $\mathbf{Q}$, the field of rational numbers. Suppose that $G$ acts on $X$ transitive$1 y$ and the action is defined over $\mathbf{Q}$. Suppose that the set of rational points $X(\mathbf{Q})$ is non-empty. Choosing $x \in X(\mathbf{Q})$ allows us to identify $G / G_{x}$ and $X$ as varieties over $\mathbf{Q}$, there $G_{x}$ is the stabilizer of $x$.

Following Ono's terminology [02], we call $G$ special if $G$ has the LeviChevalley decomposition of the form $G=U S$ over $\mathbf{Q}$, where $U$ is the unipotent radical of $G$ and $S$ is the semisimple part. We also call a homogeneous space $(G, X)$ special if $G$ and $G_{x}, x \in X(\mathbf{Q})$, are both special.

After the works of Siegel [Si] and Weil [W], Ono [ibid] defined the uniformity of a special homogeneous space $(G, X)$ in the context of integral geometry over the adele spaces attached to $(G, X)$ and introduced the Tamagawa number $\tau(G, X)$ for a special and uniform homogeneous space, and gave a criterion in terms of the homotopy groups of the complex manifold $X(\mathbf{C})$ in order that $(G, X)$ satisfies the mean value property, $\tau(G, X)=1$.

The purpose of this note is to show that any special homogeneous space is uniform and has a simple description of its Tamagawa number. It follows from some observation on the fundamental groups of algebraic groups under inner twisting and on local-global classes in $X(\mathbf{Q})$, and the fundamental result of Kottwitz $[\mathrm{K}]$ on the Tamagawa number of algebraic groups.

We wish to thank Prof. T. Ono for encouraging us to write up this note.

Notation For a field $k$ of characteristic zero, $\bar{k}$ denotes a fixed algebraic closure of $k$ and $\Gamma_{k}$ stands for the Galois group of $\bar{k} / k$.

We shall use the standard notation of Galois cohomology (cf. [Se]).

For a set $*,[*]$ denotes the cardinality of $*$

Received April 24, 1995. 


\section{Inner twisting and fundamental groups of special algebraic groups}

In this section, we prove that the fundamental groups of special algebraic groups do not change, as Galois modules, under inner twisting. Though this fact is well known for experts, we include it and give a precise proof for the sake of completeness.

Let $G$ and $G^{\prime}$ be connected linear algebraic groups defned over $k$ of characteristic zero. Suppose that $G^{\prime}$ is a $k$-form of $G$ with $\bar{k}$-isomorphism $\phi: G \rightarrow G^{\prime}$. We say that $G^{\prime}$ is an inner form of $G$ if for each $\sigma \in \Gamma_{k}$, there is $g_{\sigma} \in G(\bar{k})$ so that $\phi^{-1} \circ \phi^{\sigma}=\operatorname{Int}\left(g_{\sigma}\right)$. Note that this definition is independent of the choice of an inner twisting $\phi$.

Suppose that $G$ and $G^{\prime}$ are special (see Introduction) with the Levi-Chevalley decompositions $G=U S, G^{\prime}=U^{\prime} S^{\prime}$ over $k$, where $U, U^{\prime}$ are the unipotent radicals and $S, S^{\prime}$ are semisimple parts of $G, G^{\prime}$, respectively.

Lemma 1.1. Notation being as above, if $G^{\prime}$ is an inner form of $G$, then $S^{\prime}$ is an inner form of $S$.

Proof. Let $\phi: G \rightarrow G^{\prime}$ be $\bar{k}$-isomorphism. By definition, for each $\sigma \in \Gamma_{k}$, there is $g_{\sigma} \in G(\bar{k})$ so that $\phi^{-1} \circ \phi^{\sigma}=\operatorname{Int}\left(g_{\sigma}\right)$. Since $\phi(U)=U^{\prime}, \phi$ induces an $\bar{k}$-isomorphism $\bar{\phi}: S=G / U \rightarrow G^{\prime} / U^{\prime}=S^{\prime}$. Then, we see that $\bar{\psi}^{-1} \circ \bar{\psi}^{\sigma}=$ $\operatorname{Int}\left(\bar{g}_{\sigma}\right)$, where $\bar{x}=x U$ for $x \in G$. Hence, $S^{\prime}$ is an inner form of $S^{\prime}$.

Q.E.D

For a semisimple group $S$ defined over $k$, we define the fundamental group $\pi_{1}(S)$ to be the kernel of the universal covering of $S$ over $k$ ([O1], App. I). For a special algebraic group $G$ defined over $k$ with the Levi-Chevalley decomposition $G$ $=U S$, we set $\pi_{1}(G)=\pi_{1}(S)$, which is a $\Gamma_{k}$-module.

Lemma 1.2. Let $G$ and $G^{\prime}$ be special algebraic groups defined over $k$ and suppose that $G^{\prime}$ is an inner form of $G$. Then, we have $\Gamma_{k}$-isomorphism $\pi_{1}(G) \simeq \pi_{1}\left(G^{\prime}\right)$.

Proof. By Lemma 1.1, we may assume that $G$ and $G^{\prime}$ are semisimple. Choose an inner twisting $\phi: G \rightarrow G^{\prime}$. Take universal coverings $f: \tilde{G} \rightarrow G$ and $f^{\prime}: \tilde{G}^{\prime} \rightarrow$ $G^{\prime}$ defined over $k$. We first show that $\tilde{G}^{\prime}$ is an inner form of $\tilde{G}$. (This is in the proof of Prop. 2, Sec. 2 in [T]. But, we make things more precise here). For each $\sigma \in \Gamma_{k}$, there is $g_{\sigma} \in G(\bar{k})$ so that $c_{\sigma}:=\phi^{-1} \circ \phi^{\sigma}=\operatorname{Int}\left(g_{\sigma}\right)$. Then, $G^{\prime}$ is the twist of $G$ by 1 -cocycle $c$ valued in $\operatorname{Aut}_{\bar{k}}(G)$ ([Se], III, 1.3). Take $\tilde{g}_{\sigma} \in \tilde{G}(\bar{k})$ so that $f\left(\tilde{g}_{\sigma}\right)=g_{\sigma}$ and let $(\tilde{G})_{\tilde{c}}$ be the twist of $\tilde{G}$ by 1 -cocycle $\tilde{c}$ defined by $(\tilde{c})_{\sigma}:=$ 
$\operatorname{Int}\left(\tilde{g}_{\sigma}\right)$. Let $\tilde{\psi}: \tilde{G} \rightarrow(\tilde{G}) \tilde{c}$ be the map induced by the identity map of $\tilde{G}$ and let $f_{c}$ : $(\tilde{G})_{\tilde{c}} \rightarrow G_{c}=G^{\prime}$ be the map induced by $f$. Then, $f_{c}$ is isomorphic to $f^{\prime}$ because of the uniqueness of the universal covering ([O1], App. I) and, by our construction, $\phi \circ f=f_{c} \circ \tilde{\phi}, \tilde{\phi}^{-1} \circ \tilde{\phi}^{\sigma}=\operatorname{Int}\left(\tilde{g}_{\sigma}\right)$. Thus, our assertion follows if we check that the map $\pi_{1}(\tilde{G}) \rightarrow \pi_{1}\left(\tilde{G}^{\prime}\right)$ induced by $\tilde{\phi}$ is $\Gamma_{k}$-homomorphism. In fact, for $z \in$ $\pi_{1}(\tilde{G}), \tilde{\phi}^{\sigma}(z)=\tilde{\phi} \circ \operatorname{Int}\left(\tilde{g}_{\sigma}\right)(z)=\tilde{\phi}(z)$, since $f$ is a central $k$-isogeny.

Q.E.D.

\section{Local-global classes}

Let $G$ be a connected linear algebraic group and $X$ an algebraic variety, both defined over $\mathbf{Q}$, the field of rational numbers. Suppose that $G$ acts on $X$ transitively and the action is defined over $\mathbf{Q}$. Suppose that the set of rational points $X(\mathbf{Q})$ is non-empty. By choosing $x \in X(\mathbf{Q})$, we identify $G / G_{x}$ with $X$ as varieties over $\mathbf{Q}$, where $G_{x}$ is the stabilizer of $x$.

We introduce two equivalence relations on $X(\mathbf{Q})$. Let $y, z \in X(\mathbf{Q})$. We say that, $y, z$ are globally equivalent, written $y \sim z$, if there is $g \in G(\mathbf{Q})$ so that $z=$ gy. We say that $y, z$ are locally equivalent if there is $g_{\mathbf{A}} \in G(\mathbf{A})$ so that $z \in$ $g_{\mathbf{A}} y$, where $\mathbf{A}$ denotes the adele ring of $\mathbf{Q}$. It is easy to see that $y$ is locally equivalent to $z$ if and only if for each place $v$, there is $g_{v} \in G\left(\mathbf{Q}_{v}\right)$ so that $z=g_{v} y([\mathrm{O}]$, Lemma 6.1). Thus, the local class $\Theta_{x}$ containing $x \in X(\mathbf{Q})$ is $G(\mathbf{A}) x \cap X(\mathbf{Q})$. To study the quotient set $\Theta_{x} / \sim$, we put, for an algebraic group $A$ over $\mathbf{Q}$ or a $\Gamma_{\mathbf{Q}^{-}}$module $A$,

$$
I^{i}(\mathbf{Q}, A):=\operatorname{Ker}\left(H^{i}(\mathbf{Q}, A) \rightarrow \prod_{v} H^{i}\left(\mathbf{Q}_{v}, A\right)\right),
$$

where $v$ runs over all places of $\mathbf{Q}$.

It is known that $I^{1}(\mathbf{Q}, A)$ is finite for a linear algebraic group $A$ over $\mathbf{Q}([\mathrm{B}-\mathrm{S}])$.

The inclusion $G_{x} \rightarrow G$ induces the map $I^{1}\left(\mathbf{Q}, G_{x}\right) \rightarrow I^{1}(\mathbf{Q}, G)$. The following lemma is a refinement of [O2], Lemma 6.2.

Lemma 2.1. Notation being as above, we have a bijection

$$
\Theta_{x} / \sim \simeq \operatorname{Ker}\left(I^{1}\left(\mathbf{Q}, G_{x}\right) \rightarrow I^{1}(\mathbf{Q}, G)\right)
$$

Proof. Let $y \in \Theta_{x}$. We find $g \in G(\overline{\mathbf{Q}})$ so that $y=g x$. Since $x, y$ are invariant under the Galois group $\Gamma_{\mathbf{Q}}, y_{\sigma}:=g^{-1} g^{\sigma}$ defines a 1 -cocycle of $\Gamma_{\mathbf{Q}}$ in $G_{x}(\overline{\mathbf{Q}})$. It is easy to see that the cohomology class $c(y) \in H^{1}\left(\mathbf{Q}, G_{x}\right)$ does not depend on the choice of $g$. It was shown in [O2], Lemma 6.2 that the correspondence $y \rightarrow c(y)$ gives an injection $\varphi: \Theta_{x} / \sim \rightarrow I^{1}\left(\mathbf{Q}, G_{x}\right)$. But, it is easy to see that 
$c(y)$ becomes the trivial class in $H^{1}(\mathbf{Q}, G)$ under $G_{x} \rightarrow G$. So, we obtained an injection $\varphi: \Theta_{x} / \sim \rightarrow \operatorname{Ker}\left(I^{1}\left(\mathbf{Q}, G_{x}\right) \rightarrow I^{1}(\mathbf{Q}, G)\right)$. To show the surjectivity of $\varphi$, take $c=\left(c_{\sigma}\right) \in \operatorname{Ker}\left(I^{1}\left(\mathbf{Q}, G_{x}\right) \rightarrow I^{1}(\mathbf{Q}, G)\right)$. Then, for each $\sigma \in \Gamma_{\mathbf{Q}}$, there is $g \in G(\overline{\mathbf{Q}})$ so that $c_{\sigma}=g^{-1} g^{\sigma}$. Put $y=g x$. Then, for each $\sigma \in \Gamma_{\mathbf{Q}}, y^{\sigma}=g^{\sigma} x=$ $g c_{\sigma} x=g x=y$. Hence, $y \in X(\mathbf{Q})$ and $c(y)=c$. Further, for each place $v$ of $\mathbf{Q}$ and $\sigma \in \Gamma_{\mathbf{Q}_{v}}$, there is $g_{v} \in G_{x}(\overline{\mathbf{Q}} v)$ so that $c_{\sigma}=g_{v}^{-1} g_{v}^{\sigma}$. From this, we see that $g g_{v}^{-1}=h_{v} \in G\left(\mathbf{Q}_{v}\right)$ and $y=h_{v} x$. Hence, $y \in \Theta_{x}$.

Q.E.D

We write $h\left(\Theta_{x}\right)$ for the cardinality of $\Theta_{x} / \sim$, which is actually independent of $x \in X(\mathbf{Q})$ as we shall see below. The set $\Theta_{x} / \sim$ has a natural structure of an abelian group by the following

LEMma 2.2. Let $H$ be a special algebraic group over $\mathbf{Q}$. Then, we have bijections

$$
\begin{aligned}
I^{1}(\mathbf{Q}, H) & \simeq I^{2}\left(\mathbf{Q}, \pi_{1}(H)\right) \\
& \simeq I^{1}\left(\mathbf{Q}, \widehat{\pi_{1}(H)}\right)^{D},
\end{aligned}
$$

where $\widehat{\pi_{1}(H)}=\operatorname{Hom}\left(\pi_{1}(H), \mathbf{G}_{m}\right)$, the character module, and D stands for the Pontrjagin dual. Moreover, these bijections are functorial in $H$.

Proof. Let $H=U S$ be the Levi-Chevalley decomposition of $H$ over $\mathbf{Q}, U=$ the unipotent radical of $H, S=$ a semisimple part. Since $\pi_{1}(H)=\pi_{1}(S)$ as $\Gamma_{\mathbf{Q}}$-modules, the first isomorphism in our assertion follows from [Sa], Proposition 4.1 and Theorem 4.3. The second isomorphism is a consequence of Tate-Poitou duality. To show the functoriality in $H$, we may assume $H$ is semisimple. Then, by [B-T], Proposition. (2.24)-(i), a $\mathbf{Q}$-homomorphism between semisimple $\mathbf{Q}$-groups extends uniquely a $\mathbf{Q}$-homomorphism between their universal covering groups. This yields the functoriality in $H$ for the first bijection. For the second isomorphism, it follows from the functoriality of Tate-Poitou duality.

Q.E.D.

Proposition 2.3. Suppose that $(G, X)$ is a special homogeneous space over $\mathbf{Q}$. The number $h\left(\Theta_{x}\right)$ is independent of $x \in X(\mathbf{Q})$.

Proof. Since $G_{x}, x \in X(\mathbf{Q})$, are inner forms each other, $\pi_{1}\left(G_{x}\right)$ are all isomorphic by Lemma 1.2. Hence, our assertion follows from Lemmas 2.1 and 2.2 .

Q.E.D.

We write $h(G, X)$ for this cardinality, the number of global classes in a local 
class.

\section{Tamagawa numbers}

In this section, we assume that $(G, X)$ is a special homogeneous space over $\mathbf{Q}$, namely, $G$ and $G_{x}, x \in X(\mathbf{Q})$ are both special algebraic groups over $\mathbf{Q}$. Then, $X$ is a quasi-affine variety over $\mathbf{Q}$ so that $X(\mathbf{Q})$ is discrete in $X(\mathbf{A})$. Denote by $d G(\mathbf{A}), d X(\mathbf{A})$ the canonical measures on $G(\mathbf{A}), X(\mathbf{A})$ respectively $([\mathrm{O}], \S 4)$.

We put

$$
\tau(G)=\int_{G(\mathbf{A}) / G(\mathbf{Q})} d G(\mathbf{A})=\text { Tamagawa number of } G .
$$

We say that a homogeneous space $(G, X)$ is uniform if there is a constant $\tau(G, X)$ so that

$$
\int_{G(\mathbf{A}) X(\mathbf{Q})} f\left(x_{\mathbf{A}}\right) d X(\mathbf{A})=\tau(G, X) \tau(G)^{-1} \int_{G(\mathbf{A}) / G(\mathbf{Q})}\left(\sum_{y \in X(\mathbf{Q})} f\left(g_{\mathbf{A}} y\right)\right) d G(\mathbf{A})
$$

for any continuous function $f$ on $G(\mathbf{A}) X(\mathbf{Q})$ with compact support. When that is so, the number $\tau(G, X)$ is said to be the Tamagawa number of $(G, X)$, and we say that $(G, X)$ has the mean value property if $\tau(G, X)=1$.

Firstly, we recall the following fundamental

Theorem $3.1(\mathrm{O} 1],[\mathrm{K}],[\mathrm{C}])$. Let $H$ be a special algebraic group over $\mathbf{Q}$. Then, the Tamagawa number $\tau(H)$ of $H$ is given by

$$
\tau(H)=\frac{\left[H^{0}\left(\mathbf{Q}, \widehat{\pi_{1}(H)}\right)\right]}{\left[I^{1}\left(\mathbf{Q}, \widehat{\pi_{1}(H)}\right)\right]} .
$$

The following two theorems strengthen Lemma 8.3 and Theorem 2.1 in [02].

THEOREM 3.2. Any special homogeneous space $(G, X)$ is uniform and $\tau(G, X)$ $=\tau(G) /\left(h(G, X) \tau\left(G_{x}\right)\right), x \in X(\mathbf{Q})$

Proof. By [O], Lemma 8.3, $(G, X)$ is uniform if and only if the number $\sum_{i=1}^{h(\theta)} \tau\left(G_{x_{i}}\right)$ is independent of the local class $\Theta$, where $x_{i}, 1 \leq i \leq h(\Theta)$, stand for representatives for $\Theta / \sim$. Actually, this is the case and the equality in our assertion follows by Lemma 1.2, Proposition 2.3 and Theorem 3.1.

Q.E.D 
THEOREM 3.3. Suppose that $\pi_{1}(X(C))=\pi_{2}(X(C))=1$. Then, we have $\tau(G, X)$ $=1$, namely, $(G, X)$ has the mean value property.

Proof. By our assumption. using the homotopy exact sequence attached to

$$
1 \rightarrow G_{x}(\mathbf{C}) \rightarrow G(\mathbf{C}) \rightarrow X(\mathbf{C}) \rightarrow 1,
$$

we have

$$
\pi_{1}\left(G_{x}(\mathbf{C})\right) \simeq \pi_{1}(G(\mathbf{C})) \text { for any } x \in X(\mathbf{Q}),
$$

which is translated into $\Gamma_{\mathbf{Q}}$-isomorphism of algebraic fundamental groups:

$$
\pi_{1}\left(G_{x}\right) \simeq \pi_{1}(G) \text { for any } x \in X(\mathbf{Q}) .
$$

Therefore, $h(G, X)=1$ by Lemma 2.1 and 2.2 and $\tau(G)=\tau\left(G_{x}\right)$ by Theorem 3.1 Hence, our assertion follows from [02], Lemma 8.3 again.

Q.E.D

Theorem 3.4. Assume that $I^{1}(\mathbf{Q}, G)=1$ and let $x \in X(\mathbf{Q})$. Then, we have

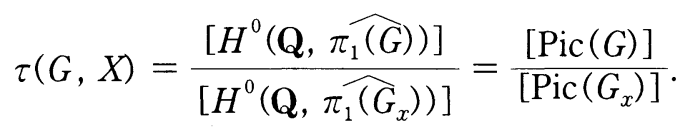

In particular, $(G, X)$ has the mean value property if and only if $[\operatorname{Pic}(G)]=$ $\left[\operatorname{Pic}\left(G_{x}\right)\right]$.

Proof. By Theorem 3.1, we have

$$
\tau\left(G_{x}\right)=\frac{\left[H^{0}\left(\mathbf{Q}, \widehat{\pi_{1}\left(G_{x}\right)}\right)\right]}{\left[I^{1}\left(\mathbf{Q}, \widehat{\pi_{1}\left(G_{x}\right)}\right)\right]},
$$

which is independent of $x \in X(\mathbf{Q})$.

By our assumption, Lemmas 2.1 and 2.2, we have

$$
h(G, X)=\left[I^{1}\left(\mathbf{Q}, \widehat{\pi_{1}\left(G_{x}\right)}\right)\right] .
$$

Since $\tau(G, X)=\tau(G) /\left(h(G, X) \tau\left(G_{x}\right)\right)$, our assertion follows from Theorem 3.1. and [Sa], Lemma 6.9. (iii).

Q.E.D.

Remark. The condition that $I^{1}(\mathbf{Q}, G)=1$ is satisfied if the semisimple part of $G$ is one of the followings. ([C] and [Sa], Theorem. 4.2, Corollary. 5.4.)

1) simply connected group,

2) adjoint group,

3) absolutely almost simple group, 
4) group which splits over a metacyclic extension.

\section{REFERENCES}

[B-S] A. Borel and J.-P. Serre, Théorèmes de finitude en cohomologie galoisienne, Comment. Math. Helv., 39 (1964), 111-164.

[B-T] A. Borel-J. Tits, Complèments a l'article 'Groupes reductifs', Publ. Math. I.H.E.S., 41 (1972), 253-276.

[C] V.I. Chernousov, On the Hasse principle for groups of type $E_{8}$. Dokl. Akad. Nauk SSSR, 306,25 (1989), 1059-1063.

[K] R. Kottwitz, Tamagawa numbers, Ann. Math., 127,3 (1988), 629-646.

[01] T. Ono, On the relative theory of Tamagawa numbers, Ann. Math., 82 (1965), $88-111$.

[02] T. Ono, A mean value theorem in adele geometry, J. Math. Soc. Japan, 20 (1968), $275-288$.

[Sa] J.-J. Sansuc, Groupe de Brauer et arithmétique des groupes algébriques linéaires sur un corps de nombres, J. reine angew., 327 (1981), 12-80.

[Se] J.-P. Serre, Cohomologie Galoisienne, Lecture Notes in Math,5, Springer (1964).

[Si] C.L. Siegel, A mean value theorem in geometry of numbers, Ann. Math., 49 (1945), $340-347$.

[T] J. Tits, Classification of algebraic semisimple groups, in Algebraic groups and Discontinuous Subgroups, Proc. Symp. Pure Math., No.9, Amer. Math. Soc., Providence, 1966.

[W] A. Weil, Sur quelques résultats de Siegel, Summa Brasil Math., 1 (1945-1946), 21-39 in OEuv. Sci., I, 339-357.

Masanori Morishita

Department of Mathematics

Faculty of Science

Kanazawa University

Kakuma, Kanazawa, 920-11, Japan

Takao Watanabe

Department of Mathematics

Faculty of Science

Osaka University

Toyonaka, Osaka, 560, Japan 